\title{
Onset and Resolution of Key Adverse Events in Valbenazine- Treated Patients with Tardive Dyskinesia: Pooled Analyses from Two Long-Term Clinical Trials
}

\author{
Stephen R. Marder, $M D^{1}$, Jean-Pierre Lindenmayer, $M D^{2}$, \\ Chirag Shah, PharmD ${ }^{3}$, Tara Carmack, $\mathrm{MS}^{3}$, \\ Angel S. Angelov, $\mathrm{MD}^{3}$ and Leslie Lundt, $\mathrm{MD}^{3}$
}

${ }^{1}$ University of California Los Angeles, David Geffen School of Medicine, Los Angeles, CA, USA, ${ }^{2}$ New York University School of Medicine, New York, NY, USA, and ${ }^{3}$ Neurocrine Biosciences, Inc., San Diego, CA, USA

Presenting Author: Stephen Marder

\section{Clinician-Reported Patient Awareness of Symptoms and Severity of Tardive Dyskinesia in Patients Prescribed VMAT2 Inhibitors}

\author{
Jonathan M. Meyer, $\mathrm{MD}^{1}$, Ericha Franey, $\mathrm{PhD}^{2}$, \\ Leslie Lundt, $\mathrm{MD}^{2}$, Betsy Benning, $\mathrm{MBA}^{3}$, \\ Edward Goldberg, MD $^{2}$, Chuck Yonan, PharmD ${ }^{2}$ and \\ Rahul Dhanda, $\mathrm{PhD}^{2}$
}

\footnotetext{
${ }^{1}$ University of California, San Diego School of Medicine, Department of Psychiatry, La Jolla, CA, USA, ${ }^{2}$ Neurocrine Biosciences, Inc., San Diego, CA, USA, and ${ }^{3}$ IQVIA, San Mateo, CA, USA
}

Presenting Author: Jonathan M. Meyer 